\title{
Ciudadanía e información en ambientes digitales
}

\author{
Héctor Alejandro Ramos Chávez*
}

Artículo recibido:

19 de octubre de 2018

Artículo aceptado:

5 de diciembre de 2018

Artículo de revisión

\section{Resumen}

En el marco de la información para la participación social en procesos políticos y democráticos, este artículo aborda el estudio de un tipo específico de ciudadanía, la cual obtiene y consulta información, pero también la genera y enriquece. Esta información va moldeando opinión pública, ciertos tipos de participación política y vinculación entre la sociedad con el gobierno. Se lleva a cabo una revisión crítica de parte de la literatura sobre el tema y se brinda una aproximación al concepto de ciudadanía digital. Se pretende generar un marco referencial sobre el estado actual del uso de internet, y sobre todo de las redes sociales digitales, como medios de consulta de información política y formación

* Instituto de Investigaciones Bibliotecológicas y de la Información, UNAM, México

aramos@iibi.unam.mx

INVESTIGACIÓN BIBLIOTECOLÓGICA, vol.33, núm.78, enero/marzo, 2019, México, ISSN: 2448-8321 pp. 143-163 
de opinión pública. Se concluye advirtiendo que, si bien las tecnologías de la información y comunicación brindan mecanismos interesantes para la consulta y generación de información para la construcción de ciudadanía digital, existen elementos que se deberían tomar en consideración, pues están relacionados con la calidad y veracidad de la información que está utilizando la ciudadanía digital para involucrarse con el devenir de los asuntos públicos.

Palabras clave: Información; Internet; Ciudadanía digital; Redes Sociales Digitales; Opinión Pública; Participación Cívica

\title{
Information in digital spaces for the construction of citizenship
}

Héctor Alejandro Ramos Chávez

\begin{abstract}
Within the scope of information for social participation in political and democratic processes, this article addresses the study of one specific type of citizenship; one that not only obtains and consults information, but also generates and enriches it. This type of information shapes public opinion as well as certain types of political participation and connection between society and government. The article conducts a critical literary review on the subject matter and provides an approximation of the concept of digital citizenship. The article attempts to provide a referential framework on the current status of internet usage-and particularly digital social networks-as a means of consulting political information and forming public opinion. The article concludes by cautioning that, while information and communication technologies offer interesting mechanisms for consulting and creating information to build digital citizenship, there are issues that should considered relating to the quality and veracity of the information that the digital citizenry is using to be involved in the development of public issues.
\end{abstract}

Keywords: Information; Internet; Digital Citizenship; Digital Social Networks; Public Opinion; Civic Participation 


\section{INTRODUCCIÓN}

-1 l surgimiento y la configuración de una sociedad cada vez más vincuElada con las tecnologías de la información y la comunicación (TIC) han modificado el entendimiento y la forma de estudio de diversas disciplinas científicas. Ejemplo de ello se refleja en el campo de la ciencia política, debido a la importancia actual de las redes sociales digitales, también conocidas como redes sociodigitales (Trejo-Delarbre, 2017), definidas como escenarios del discurso político y formación de opinión pública (McClurg, 2003; Watts y Dodds, 2007); en la sociología, por las inéditas formas de interacción social (Beck, 1998; Beck y Beck-Gernsheim, 2003; Castells, 2002, 2006; La Due Lake y Huckfeldt, 1998; Wellman et al., 2001); en la antropología, por los nuevos escenarios de representación cultural e intercambios simbólicos, culturales y sociales (Rodríguez, 2005; Shields, 1996); y por supuesto, en la bibliotecología y los estudios de la información, dados los recientes procesos para obtener, consultar, generar, enriquecer y resguardar información que representa el uso de estas tecnologías (Bertot, Jaeger y McClure, 2010; Morales Campos, 2006; Voutssas, 2006, 2017).

En este sentido, el problema de investigación de este trabajo consiste en la necesidad de entender y debatir los procesos, mediados por las tecnologías digitales, por los cuales los individuos están formándose opinión pública y, en algunos casos, llevando acción colectiva en miras de atender asuntos de interés público. Interesa particularmente entender el papel específico de las redes sociales digitales como espacios de obtención de información y formación de opinión relacionada con los asuntos públicos.

El método utilizado es el deductivo documental, mediante la revisión de trabajos que abordan tanto la cuestión de ciudadanía en general como de aquellos que analizan a la ciudadanía digital de forma particular. De igual forma, se utiliza un método heurístico que tiene por objetivo estabilizar el uso del concepto de la ciudadanía digital con relación a la información también contenida en los medios digitales.

La delimitación del trabajo estará determinada por la revisión del estado del arte del concepto de ciudadanía digital, tomando preferentemente aquellos autores que vinculen en su definición la idea de la información. De igual forma, se hará énfasis en el contexto latinoamericano, específicamente en la situación mexicana.

Cabe hacer hincapié que, con el uso de las tecnologías, los individuos están obteniendo, pero también generando y enriqueciendo información que les permite formarse opiniones y tomar decisiones de carácter individual y colectivo. En ese sentido, en este trabajo interesa de forma particular conocer, 
entender y debatir las formas, mediadas por las TIC, por las cuales los individuos consultan, producen y enriquecen la información, a la par de entender el impacto que estos mismos procesos están teniendo en la formación de un tipo específico de ciudadano, propuesto por algunos autores como ciudadano digital (Benítez, 2013; Campos y Silván, 2012; Natal, Benítez y Ortiz, 2014; Ribble, Bailey y Ross, 2004; Robles, 2009; Shelley et al., 2004; Sterret, 2012, entre otros).

En este punto, se considera pertinente dividir este artículo en cuatro apartados generales. En el primero de ellos se abordará la temática del entendimiento del concepto de ciudadanía, con objeto de establecer las bases para el posterior entendimiento específico de la ciudadanía digital. En el segundo apartado se pondrán en consideración una serie de estudios que han analizado las tendencias en las que la ciudadanía está utilizando las tecnologías de la información y comunicación para informarse y formarse opinión pública, para lo cual se tomarán en cuenta estudios internacionales y algunos específicos de la situación en México. En el tercer apartado se abordarán algunas de las características específicas de la Red, así como algunos elementos que adquiere la información en línea, además de enunciar algunas de las problemáticas que se deberían tomar en consideración en el análisis. Se concluirá con algunas reflexiones y se propondrán algunos derroteros de investigación futura.

\section{LA TRANSICIÓN DE LA CIUDADANÍA A LA CIUDADANÍA DIGITAL}

No resulta ocioso plantear, en un primer momento, un entendimiento general sobre el concepto mismo de ciudadanía, para posteriormente entender de mejor forma el concepto específico de ciudadanía digital y los procesos que están marcando la obtención de información, la formación de opinión pública y los procesos de interacción social y acción colectiva en ese tipo específico de ciudadanía.

En primer lugar, se debe entender que el concepto de ciudadanía ha tenido importantes cambios a lo largo de la historia, por lo que no podemos entenderla como una noción estática, sino en constante evolución y reconceptualización. En ese sentido, su entendimiento ha pasado del análisis exclusivo de los derechos y obligaciones atribuidas a los individuos a un entendimiento más amplio, referente a la capacidad de los ciudadanos de informarse y participar constantemente en los asuntos públicos y la forma en que son abordadas y resueltas las problemáticas sociales. 
Igualmente, resulta útil resaltar la hipótesis sociológica del ciudadano como individuo perteneciente a una comunidad de iguales, donde se establecen principios y derechos que permiten alcanzar esa igualdad. Esos derechos van desde el principio de igualdad de trato por parte de las autoridades públicas a todos los ciudadanos, hasta el disfrute de los beneficios propios del sistema económico, relacionados con las posibilidades de ganar, ahorrar, comprar, rentar, etc. Sin embargo, gran parte de las discusiones del concepto de ciudadanía, más que a destacar estos puntos referenciales del concepto, apuntan a resaltar las desigualdades existentes entre los individuos en términos de propiedad, de trato, de desarrollo de capacidades y oportunidades de los ciudadanos (Torres, 2007: 33).

De igual forma, con objeto de contextualizar el uso del concepto de ciudadanía, conviene brindar algunas nociones clásicas en su entendimiento. En primer lugar, Marshall (1997) divide a la ciudadanía en tres partes o elementos: el civil, el político y el social, que evolucionaron, para el caso inglés, en el disfrute de los derechos cívicos (alcanzados en el siglo XVIII), de los derechos políticos (del siglo XIX) y de los derechos sociales (del siglo XX). Esta visión de ciudadanía, aunque sienta las bases de su uso conceptual, fue criticada principalmente por la supeditación que se aprecia del ciudadano a las decisiones públicas gubernamentales (Turner, 1990). Lo anterior dio paso a que otros autores vincularan con mayor ahínco el tema de la democracia con el tema de la ciudadanía, dando como resultado aspectos que en la actualidad son fundamentales al abordar el tema de la ciudadanía. Algunos de estos elementos son la esfera pública, la identidad política, la solidaridad social, la autonomía, el reconocimiento a la diferencia, la cultura política, la acción social y la participación ciudadana (Vieira, 1998). Lo anterior se acerca al tercer elemento relacionado con las actividades de la humanidad en la tierra descritas por Arendt (1993). En este sentido, esta autora menciona en primer lugar la existencia de un Homo faber, vinculado al mundo de trabajo pues es el hombre que hace o fabrica; un Homo ludens, relacionado con el mundo del placer pues es el hombre que juega o está interesado en la diversión; y finalmente, que es donde podríamos ubicar a la ciudadanía, a un Homo sapiens, que es aquel hombre que piensa y está vinculado con la idea de Habermas (1987) del mundo de la vida. Este mundo de la vida es el espacio donde, mediante la deliberación y la argumentación de ideas, el individuo aparece en el escenario social mediante la lógica de la acción comunicativa.

Tomando en consideración esto, en este trabajo se parte de la idea de la ciudadanía como la condición de reconocimiento de los individuos en su carácter público que, por su pertenencia a una comunidad determinada, les permite ejercer derechos políticos, sociales y económicos, así como la capacidad 
de intervenir en el desarrollo e incidir en el rumbo de la política de esa comunidad. Se debe también advertir de las desigualdades y las situaciones de vulnerabilidad y atraso de una parte importante de la sociedad y cómo ésta lleva a cabo los elementos atribuidos a la idea del ejercicio ciudadano.

Podemos ubicar una variedad amplia de formas de ciudadanía que toman en consideración desde el grado de participación hasta el tipo y las características de esa misma participación. En ese sentido, la ciudadanía puede diferenciarse, en primer lugar, como ciudadanía pasiva (representativa, delegativa o de baja intensidad) o ciudadanía activa (deliberativa, participativa o de alta intensidad). La primera da cuenta de un ciudadano que, aun ejerciendo su derecho a elegir representantes públicos y cumpliendo con sus obligaciones ciudadanas, se mantiene al margen de la participación del desarrollo de los asuntos públicos. Por el contrario, una ciudadanía activa se relaciona con un ciudadano que, a la par de cumplir con las características anteriores, también se informa constantemente e interviene en el desarrollo de los asuntos y la actividad pública.

En cualquiera de estas formas de ciudadanía, la información juega un papel central en la forma en que el individuo se relaciona con los asuntos públicos. Desde una ciudadanía pasiva se requeriría de información para conocer los derechos y las obligaciones ciudadanas, así como de información relacionada con los candidatos a ocupar cargos de representación popular, con objeto de ejercer responsablemente el derecho al voto. Mayor relevancia adquiere el tema de la información en la idea de una ciudadanía activa, pues ésta requeriría de una información constante sobre la forma en la que están siendo atendidos los problemas y asuntos públicos, y con ello poder participar en su tratamiento.

De igual forma, se puede hacer una diferenciación dependiendo de las características que tenga la participación ciudadana. Ésta podría ser administrativa o política, e institucional o autónoma, también referida por algunos autores como convencional o no convencional (Natal, Benítez y Ortiz, 2014: 198). La ciudadanía administrativa se refiere a aquélla en la que los individuos ejercen sus derechos y obligaciones de carácter procedimental con la administración pública, sin que se ponga en entredicho la gestión gubernamental; por otro lado, la ciudadanía política sí cuestiona el actuar gubernamental e intenta modificar las formas de gobierno. La participación ciudadana institucional (o convencional) es aquélla que usa los canales institucionalmente establecidos para participar, mientras que la autónoma (o no convencional) se refiere a la participación no mediada por los canales institucionales y que puede surgir de la organización social y la acción colectiva.

Estas mismas características han tenido una réplica en las formas específicas de la participación ciudadana mediada por las TIC, y han confluido 
en denominar la formación ciudadana actual como ciudadanía digital, con variantes como ciudadanía electrónica (Dader, 2003; Silva, 2010), ciberciudadanía (Álvarez, 2009; Pérez, 2014) y e-ciudadanía (Griffiths, 2004; Kaufman, 2005). En este sentido, se ha propuesto entender a la ciudadanía digital desde diversas perspectivas. En primer lugar, desde un plano administrativo-institucional, al definirla como "aquellos ciudadanos que ejercitan todos o algunos de sus deberes y derechos a través de comunidades virtuales" (Robles, 2009: 37). En segundo lugar, desde un plano mucho más participativo e involucrado con el desarrollo de los asuntos públicos, vinculada más con un plano político-autónomo, al caracterizarla como el "conjunto de prácticas políticas y ciudadanas que de una forma u otra tratan de modificar y/o incidir en las instituciones, a través del uso de medios y tecnologías que tienen como característica la digitalización de sus mensajes y contenidos, como por ejemplo internet, pero también con otras tecnologías de información y comunicación (las llamadas TIC)" (Natal, Benítez y Ortiz, 2014: 9). Al margen de estas interpretaciones, en lo que parece haber un acuerdo general es en definir a la ciudadanía digital como un ejercicio del individuo "capaz de ejercer su ciudadanía bajo un panorama de interactividad ofrecido por una plataforma digital" (Arcila, 2006: 18).

Por todo lo anterior, se considera pertinente el entendimiento de ciudadanía digital como la parte de la sociedad que, además de ejercer sus derechos y cumplir con sus obligaciones enmarcadas en la idea clásica de ciudadanía, lleva a cabo procesos de información, organización y participación social mediante la utilización de las tecnologías digitales.

En este sentido, existen muchas maneras en las que los ciudadanos se informan y llevan a cabo procesos novedosos de participación política que, al surgir en un modo online, pasan, en algunas ocasiones, al mundo offline. Estas formas para obtener información y participar tienen que ver desde el uso de las aplicaciones más populares, como los wikis, Facebook, Twitter, Instagram y Youtube, hasta herramientas más sofisticadas, muchas de las cuales tienen el propósito de mejorar la calidad de la información que se consulta, así como mejorar los procesos para la discusión, el intercambio de ideas y la llegada a acuerdos colectivos, como las aplicaciones Reddit, Appgree, Loomio y Agora Voting System. Estas aplicaciones están siendo utilizadas como medios para la obtención, consulta, enriquecimiento y generación de información, pero también como medios para el análisis político y social, pues se están perfilando cada vez más como lugares de acceso a la información, encuentro social, debate, generación de opinión pública y espacios de presión política que puede generar capital social y acción colectiva.

Por lo tanto, un elemento que definiría el que se sea o no ciudadano digital estaría determinado por el uso de las tecnologías de la comunicación 
como medios para la obtención de información socialmente relevante, pero también como espacios de encuentro social y lugar de intercambio y debate de las ideas sobre los asuntos de interés público. En este sentido, el uso de las tecnologías con fines exclusivamente lúdicos, de ocio, entretenimiento o diversión no se vincularían con la noción de ciudadanía digital, pues no se vería en esos usos un aspecto social amplio (más que los meramente individuales o grupales) y no existiría una vinculación con temas políticos o de interés general en términos sociales. Igualmente, necesario en este análisis resultaría, desde el plano institucional y en un entendimiento de gobierno abierto, la existencia de espacios digitales para la participación ciudadana digital (hablando desde el plano de la ciudadanía digital institucional), en donde se deberían tomar en consideración esfuerzos gubernamentales incluidos en los debates de e-government, Open government y data government. Estos mismos esfuerzos están tomando mayor fuerza en muchos países al incluir en las agendas de gobierno dos elementos muy importantes: 1) remarcar el derecho insoslayable al acceso a información pública gubernamental, que conlleva transparencia y rendición de cuentas gubernamentales, y 2) la necesidad de incluir la voz de los ciudadanos en la gestión y tratamiento de los asuntos públicos. Estos elementos pueden ser potenciados por el uso de las tecnologías por parte del gobierno, pero también por parte de una ciudadanía digital cada vez más vinculada con los asuntos de interés público.

\section{TENDENCIAS EN EL USO DE INTERNET PARA INFORMARSE Y FORMARSE OPINIÓN PÚBLICA}

Algunos de los estudios que han analizado las tendencias sobre el uso del internet destacan la forma en la que la ciudadanía lo está utilizando para informarse sobre temas de actualidad en términos políticos, sociales y económicos (Aparicio, 2011; Asociación de Internet.mx, 2018; Asociación para la Investigación de Medios de Comunicación, 2017; Hintlin, 2018; Horrigan y Duggan, 2015; Marcos, Sánchez y Olivera, 2018; Sánchez Navarro y Aranda Juárez, 2011; Zickuhr, 2013). De igual forma, estos estudios están demostrando que internet, en específico las redes sociales digitales, han desplazado a los medios de comunicación tradicionales que usualmente utilizaban los individuos para informarse, como la radio, la televisión o la prensa. En ese contexto, la generación de contenidos y la búsqueda de información se ha facilitado con el uso de las tecnologías y en específico de las redes sociales digitales, y se ha mencionado que de de contenidos. No sólo les interesa el entretenimiento. La información, los 
servicios, compartir ideas y proyectos, mejorar la vida de las personas; en definitiva, diversificar para que los usuarios de las redes encuentren todo aquello que antes les ofrecía una biblioteca y más. (Marcos, Sánchez y Olivera, 2018: 736)

En algunos estudios a nivel internacional se ha demostrado que, en países como Estados Unidos de América, aunque en el pasado más cercano se registró un rápido crecimiento en el número de usuarios de las tecnologías digitales, en los últimos dos años se ha mantenido estable el número de estadounidenses que se conectan, usan las redes sociales digitales o son dueños de algún dispositivo para conectarse (computadora, laptop, tableta, teléfono celular, etc.) (Hintlin, 2018).

Lo anterior ha demostrado, quizá, que se ha llegado a una cobertura muy amplia de las personas adultas que usan, o están interesadas en utilizar, las tecnologías. En este sentido se ha mencionado que un factor que contribuye a la desaceleración del crecimiento en esas áreas es que amplios sectores de la población han alcanzado niveles saturados de adopción de las tecnologías (Horrigan y Duggan, 2015).

Aun con esos datos, siguen existiendo sectores de la población que no se conectan a internet. Esto no obedece necesariamente a temas de vulnerabilidad o a situaciones de marginalidad, sino que se relaciona con aquellos sectores de la población que no les interesa la utilización de estas tecnologías por no considerarlas importantes en sus vidas. En este sentido, estudios como el de Zickuhr (2013) han intentado demostrar cuáles son los factores que contribuyen a que los no usuarios de internet no lo sean. De forma puntual, son principalmente aspectos relacionados con la importancia de internet o lo difícil de su empleo lo que representa en mayor grado su no uso con el $66 \%$ de las menciones, sólo 26 \% se refiere a aspectos relacionados con los costos de internet (Zickuhr, 2013).

Otro caso interesante es el de España, donde algunos estudios (Asociación para la Investigación de Medios de Comunicación, 2017; Sánchez Navarro y Aranda Juárez, 2011) han mostrado la importancia del uso de internet como herramienta fundamental, en la actualidad, para la búsqueda y obtención de información para el sector juvenil de ese país. Sánchez Navarro y Aranda Juárez (2011) toman en consideración tres dimensiones de análisis (1. usos, 2. percepciones, y 3. uso específico de redes sociales), y han mostrado que los principales usos de las redes sociales digitales son los usos de amistad, con $79.5 \%$, y de contacto con personas con $66.6 \%$. Sobre los usos de estas mismas plataformas para la búsqueda de información, apenas $27.8 \%$ de menciones se relacionan con la consulta o solicitud de información sobre algún tema de su interés. 
Estos resultados contrastan, de cierta forma, con los resultados de los usos específicos que se le da a la Red para consultar información en aquel país. En el estudio de 2017 de la Asociación para la Investigación de Medios de Comunicación (AIMC), se mostró la importancia de internet para informarse en España, pues los datos arrojaron que ocho de cada 10 personas consultan internet para mantenerse informados sobre los asuntos relacionados al ámbito noticioso.

Para el caso de México, algunos estudios brindan datos muy interesantes que ilustran la importancia que está adquiriendo el análisis de este tipo de formas de obtención de información por parte de la ciudadanía. Ejemplo de ello son los resultados del Estudio sobre los hábitos de los usuarios de internet en México, en su edición de 2018, donde se hace mención, entre otras temáticas, a que los usuarios de internet en México han aumentado de 20.2 millones de usuarios en 2006 a 70 millones de usuarios en 2016 (Asociación de Internet.mx, 2018).

Tomando en consideración las actividades que los individuos llevan a cabo al utilizar internet, resalta, en primer lugar, la revisión y consulta de sus redes sociales digitales, con $83 \%$ de menciones; el envío o consulta de correos recibidos con $78 \%$; enviar o recibir mensajes instantáneos (chats), $77 \%$; la búsqueda de información constituye el cuarto mayor uso de internet con $74 \%$. Estos resultados son muy ilustrativos sobre la importancia que está adquiriendo el uso de internet en la actualidad para informarse e intercambiar información a través de las redes sociales digitales.

Otro aspecto interesante de este estudio es la vinculación que realiza sobre las temáticas del uso de internet y la democracia en el país. En este sentido, $92 \%$ de los entrevistados mencionó la búsqueda de información relacionada con los procesos electores como un elemento muy importante que les ha servido para ejercer o mejorar sus derechos democráticos.

Sin embargo, debe tomarse en consideración que no por el hecho de estar mejor informados existe necesariamente mayor participación política o social por parte de los individuos. Se ha alertado que

en general, más información más descentralizada es mejor que menos. Y la información barata también reduce los costos de coordinación de una acción colectiva. Pero ¿qué tipo de información demanda la ciudadanía? A juzgar por los contenidos de los estanquillos de periódicos y revistas, así como por los trending topics de Twitter, la demanda por información políticamente relevante es la excepción a una regla sencilla: la frivolidad siempre es más interesante que la polaca. (Aparicio, 2011:205) 
Desde esta perspectiva convendría también detenernos a analizar el tipo de información política con base en la cual los ciudadanos se están formando opinión pública y generando, en su caso, acción colectiva.

Ahora bien, con relación a los lugares en donde se está buscando la información, sobresalen las redes sociales digitales con $97 \%$ de incidencia, superando con ello inclusive a los portales especializados de noticias (páginas de noticieros, diarios, semanarios, etc.), con $79 \%$; de igual forma, los motores de búsqueda son importantes para este proceso de búsqueda de información, con $74 \%$; además de la visita de blogs, foros, sitios de videos/podcasts y publicidad política en línea, todos ellos con menor porcentaje de uso (Asociación de Internet.mx, 2018). Esta utilización tan amplia de las redes sociales digitales como lugares para obtener información política obliga a repensar las características específicas que adquiere la propia información en línea, así como las potencialidades y las limitantes posibles en su uso.

Este tipo de estudios, a manera de análisis experimentales, han brindado elementos muy interesantes para intentar conocer y cuantificar los usos no solamente sociales, sino sobre todo de utilización política, democrática y participativa que se le da a la información consultada en internet, de forma específica difundida a través de las redes sociales digitales. Una vez tomados en consideración estos elementos, en el siguiente apartado de este trabajo se abordarán las principales características de la información consultada en internet, así como algunos derroteros de su análisis.

\section{CARACTERÍSTICAS, POTENCIALIDADES Y PELIGROS DEL USO DE INTERNET PARA LA FORMACIÓN DE OPINIÓN PÚBLICA}

Una vez tomado en consideración el análisis de cómo los individuos están utilizando internet y en específico las redes sociales digitales para obtener y generar información en términos de interés público, conviene abordar de forma específica las características que adquiere la información con estas tendencias, así como dar cuenta de las potencialidades que conlleva el uso de las tecnologías, pero también de las limitantes así como las problemáticas que se deberían tomar en consideración en el análisis.

En primer lugar, con respecto a las características que está adquiriendo esta forma de ciudadanía, se han destacado algunas (Natal, Benítez y Ortiz, 2014) que de cierta forma se diferencian de las formas tradicionales de obtención de información, por lo que resultan interesantes para tomar en cuenta, así como profundizar en su análisis para acercarnos a una comprensión más 
amplia de la ciudadanía digital. En un primer momento se podría señalar la inmediatez en la producción y transmisión, así como la recepción de información, como una primera característica de la Red que ha impactado la forma en la que se transmite información en los medios digitales. Esta característica brinda la posibilidad de disponer y acceder a la información casi de manera inmediata a la sucesión de los eventos o generación de la propia información, por lo que se puede acceder de una forma más veloz a ella tanto en el lugar en el que nos encontremos o bien en otras latitudes. Con esta característica se ha mencionado que han desaparecido, o por lo menos se han difuminado, las barreras espaciotemporales que enfrentaba la transmisión de información con anterioridad al uso de las tecnologías (Monfort, 2013). De igual forma, la propia inmediatez les permite a las personas tener un contacto más directo tanto con la información como con otras personas ubicadas alrededor del mundo; contacto que sería muy costoso mantener por otros medios. En otras palabras, esta inmediatez les "permite a los usuarios de las TIC tener una relación mucho más directa con otros usuarios y con la información misma” (Natal, Benítez y Ortiz, 2014: 11).

Otra característica de la información online es la interactividad, entendida como la capacidad de los individuos de obtener un abanico más amplio de interacción en términos cualitativos y cuantitativos con la información. De esta forma, la obtención de información digital permite no circunscribirla con exclusividad a textos, sino a amplias posibilidades multimedia, como la de crear, consultar, enriquecer e intercambiar información en formatos sonoros, audiovisuales e incluso fotográficos (Cabero, 2007). Esta interactividad de la información les permite a los ciudadanos mayores herramientas y posibilidades de expresar sus ideas y participar en los asuntos públicos, con lo cual se rompe la pasividad ciudadana en su involucramiento con lo público (Kymlicka y Norman, 1997). De igual forma, con esta misma característica se ha permitido generar "otras modalidades informativas, otros modos de narrar los datos, los hechos, las ideas y opiniones, otros recursos expresivos que superan la integración audio-escrito-visual de los medios anteriores hasta constituir un lenguaje multimedia" (Cebrián, 2009: 16).

Una tercera característica, vinculada de cierta forma con la interactividad, es la multiautoría de la información y de los contenidos de internet. Con esta particularidad el ciudadano tiene la posibilidad de que, a la par de poder consumir información, también se le abre la posibilidad de generarla, modificarla o enriquecerla; es decir, a la par de consumidor, puede ser productor y enriquecedor de información. Este rasgo, que puede verse de manera amplia en la creación y desarrollo de wikis, rompe la visión anterior de información, la cual tenía como distintivo la forma vertical y unidireccional 
del proceso generación-obtención de información. Con esta característica, inclusive, se ha hablado de un nuevo tipo de individuo bajo el concepto de prosumidor (Dezuanni y Monroy, 2012; Islas, 2010). Éste hace referencia a los ciudadanos que, al mismo tiempo que consumidores, son también creadores de información y contenidos en línea. El concepto de prosumidor va más allá al incluir la capacidad de interacción entre diversos ciudadanos con miras a generar acción colectiva (Postmes y Brunsting, 2002).

Otra característica es la accesibilidad, que representa el costo relativamente bajo de consulta y obtención de información por internet con respecto a otras formas para obtenerla. Gracias a esto se abre la puerta a individuos a los que de otra manera les resultaría muy difícil acceder a la información y a la comunicación con grupos más amplios para el enriquecimiento de conocimientos y generación de opinión pública (Natal, Benítez y Ortiz, 2014). En este punto conviene detenerse a analizar nuevamente los problemas tanto de desigualdad de acceso a ciertos recursos como de capacidades para sacarles provecho que ponen en desventaja a ciertos sectores de la población para el disfrute y uso de estas tecnologías.

Una última característica es la libertad de expresión como resultado de hacer uso de estas tecnologías para emitir comentarios y formas de pensar sobre cómo son tratados los asuntos públicos. En este sentido, la producción de información y participación en internet puede darse de forma más natural, sin miedo a la censura y a los juicios que los contenidos y comentarios emitidos puedan generar. Aunque también se debería tomar en consideración la problemática, cada vez más recurrente, que representa el manejo de los datos personales, pues la intimidad de estos ha estado cada vez más en entredicho por los problemas de vulnerabilidad y uso incorrecto.

Asimismo, algunas características específicas de la información puesta a disposición en línea no recaen necesariamente en el plano de las virtudes cívicas o democráticas. En este sentido, una de las críticas que en la actualidad ha cobrado mayor fuerza es el uso del discurso político que no recae en la verdad, con objeto de realizar llamamientos más al aspecto emocional que a una realidad concreta en el tratamiento de la información. Si bien este fenómeno fue puesto a debate mediante el término posverdad desde 1992 por Tesich, se ha visto magnificado por el uso de las tecnologías como medios para obtener información. Es decir, ahora los políticos utilizan las mismas herramientas digitales para generar discursos no fundamentados en la realidad, y estos discursos están generando opinión pública.

De igual forma, con temas como el de las noticias falsas o alternativas, la calidad de la información consultada en línea, y sobre todo en las redes sociales digitales, debería pasar por un proceso de verificación por parte del 
usuario. Sin embargo, esta verificación rara vez se lleva a cabo, lo que da como resultado que esa misma información falsa esté también teniendo impacto en la construcción de ciudadanía. Este fenómeno puede extenderse más, pues muchas veces estas noticias falsas o alternativas son difundidas indiscriminadamente por muchos usuarios.

Derivado de estas características y las potencialidades de internet como medio para informarse y participar, se puede apreciar que la ciudadanía digital podría estar determinada, en un primer momento, por ser o no usuario de las tecnologías y principalmente internet (Robles, 2009). Por lo que, para una extensión más amplia de su entendimiento como un derecho para toda la sociedad, se debería también tomar en consideración a todo aquel segmento de la población que, por problemas económicos, de infraestructura o de ubicación geográfica están marginados de estas posibilidades. En este punto surge la necesidad de tomar en cuenta el problema de la brecha digital (Castells, 2001; Hoffman, Novak y Schlosser, 2001; Rodríguez, 2006; Servon, 2002), y más aún si se considera el contexto de México y la región latinoamericana, que ha sido denominada la región más desigual del mundo (Burchardt, 2012; Fleury, 2005; ONU, 2012; OEA, 2014).

En este sentido, se puede definir a la brecha digital como la disparidad entre los que tienen y no tienen acceso a internet (Castells, 2001). Esta disparidad puede deberse a situaciones de carácter económico, pero también de capacidades necesarias para usar las tecnologías, tema vinculado con los debates de las habilidades digitales (Burin et al., 2016) y de la alfabetización informacional (Bruce, 1999; Spitzer, Eisenberg y Lowe, 1998).

Aunado a la brecha digital, convendría hacer referencia a algunos de los problemas que se han encontrado en la utilización de estas herramientas como medios para la información y la participación colectiva, con objeto de no quedarnos exclusivamente en el plano idílico del concepto de la ciudadanía digital. Se han encontrado problemáticas relacionadas con la influencia virtual (Nielsen, 2006); las diferencias socioeconómicas que afectan el uso de las tecnologías (Sterret, 2012); la escasa o nula reglamentación y legislación en materia de internet (Vleugels, 2009); el uso con propósitos de ocio sobre el uso ciudadano que se le da a la Red (Griffiths, 2004); el progresivo uso de internet con fines de propaganda política (Campos y Silván, 2012); la saturación informativa (Palanque y Paternò, 1998) y la misma calidad de la información en línea (Fernández-Ramos, 2015), entre otras problemáticas que se deberían tener presentes en una investigación de esta naturaleza.

Tomando en consideración estas perspectivas, resulta pertinente adentrarse en el estudio sobre cómo las personas, en la actualidad, se están informando, formando opinión pública y ejerciendo su ciudadanía a partir de 
procesos participativos en ambientes digitales mediante la generación de acción colectiva y capital social, o si, por el contrario, se están generando procesos de individualización y fragmentación social.

De igual forma, el tema de las potencialidades de las bibliotecas digitales toma interés en estos estudios, pues la biblioteca, en términos generales y entendida como institución que resguarda y facilita el acceso a la información, también puede ser de gran utilidad para el acceso a información de la ciudadanía con objeto de fomentar la integración y participación ciudadana utilizando también las herramientas digitales como internet, y de forma específica, las redes sociales digitales. Estos elementos podrían ayudar a superar las problemáticas relacionadas con esa parte de la ciudadanía renuente e indiferente a participar en los temas de interés público y en los compromisos de interés social y político, pues el primer paso para fomentar una ciudadanía más participativa, deliberativa y de alta intensidad es contar con el hábito permanente de la documentación y la información. En este punto, los sistemas bibliotecarios, entendidos como unidades documentales de los sistemas de información de las naciones, pueden considerarse recursos vitales para potenciar las capacidades ciudadanas de deliberación, encuentro y compromiso cívico.

En este mismo contexto, resulta útil tomar en consideración las iniciativas en materia de desarrollo de servicios bibliotecarios digitales, la generación de catálogos electrónicos de bibliotecas, las iniciativas para promover las habilidades y destrezas digitales para la búsqueda de información, así como las capacidades de las bibliotecas digitales para la identificación de necesidades de información de diversas comunidades. Estas temáticas se constituyen como elementos fundamentales para potenciar el vínculo entre la biblioteca y la ciudadanía, sobre todo la ciudadanía digital. En este sentido, la investigación sobre las Smart Libraries (Miller et al., 2004; Repanovici et al., 2009; Sebestova et al., 2014; Won Min, 2012) está cobrando interés en círculos especializados de análisis, al intentar ubicar esas potencialidades que pueden promoverse desde las bibliotecas digitales para fomentar las capacidades ciudadanas de intervención continua en el desarrollo y rumbo de los asuntos públicos.

\section{Conclusiones}

La utilización de las tecnologías de la información y la comunicación, sobre todo internet, han permitido dejar atrás formas verticales de obtención de información, en donde los medios tradicionales de comunicación se constituían como los más importantes espacios para la formación de opinión 
pública. Según los datos abordados en este trabajo, cada vez más ciudadanos utilizan internet, y sobre todo las redes sociales digitales, con objeto no sólo de buscar y consultar información, sino también para generarla y enriquecerla.

Esta utilización de las tecnologías podría impactar en dos niveles de análisis. Por un lado, con un enfoque top-down, el gobierno puede utilizar estas herramientas para abrir espacios de comunicación e interrelación con los ciudadanos y con ello profundizar en valores democráticos como la transparencia, la rendición de cuentas y la inclusión ciudadana. Por otro lado, ahora con un enfoque bottom-up, los ciudadanos cuentan con un instrumento muy valioso que les permite, a la par de informarse, generar presión social y política al cauce que va adquiriendo el tratamiento de los asuntos públicos.

En este contexto se está perfilando un tipo de ciudadanía ligada a la utilización constante de estos canales, denominada ciudadanía digital, la cual a la par de formarse opinión pública mediante la utilización de internet va generando novedosos espacios de encuentro cívico y lugares de debate y deliberación sobre los asuntos de interés público. Lo anterior resulta oportuno para el incremento de espacios para la consulta de información e intercambio de opiniones, y conlleva la necesidad de revisar algunos elementos para conocer con base en qué la ciudadanía genera participación y acción colectiva. En este mismo punto, se está convirtiendo cada vez más en lugar común hacer referencia a los aspectos negativos de la utilización social de estos medios, discusiones que van desde el fenómeno de la posverdad, profundizada fuertemente por la utilización de internet y las redes sociales, hasta aspectos como las fake news, las noticias alternativas o el Cambridge Analytica, relacionado con la seguridad de los datos personales en las redes sociales y el envío de información "a modo" para sesgar o parcializar las posturas de los usuarios de las mismas redes. Tales elementos, entre otros, se deberían tomar en consideración para conocer realmente la calidad y veracidad de la información con la que la ciudadanía digital genera opinión pública y realiza acción colectiva.

Lo anterior no resulta un tema menor ni periférico, al contrario, se perfila como uno de los elementos que debemos abordar con mayor importancia. Gran parte de la información disponible en internet carece de una calidad y una veracidad mínimas, lo que desemboca a su vez en una baja calidad en la fundamentación de la opinión pública. Desde estas perspectivas, y a la par de la importancia de generar una cultura de la verificación de la información que se encuentra en internet, en específico en las redes sociales digitales, también resulta oportuno debatir sobre las herramientas con las que cuentan los ciudadanos para verificar la veracidad y la calidad de la información que están consultando. 
En este sentido, los servicios digitales puestos en marcha por las bibliotecas públicas podrían jugar un papel fundamental para mejorar la calidad y certeza de la información que está siendo utilizada por la ciudadanía. En este punto, servicios y actividades en materia bibliotecológica como el desarrollo de colecciones digitales, la generación de catálogos electrónicos, los cursos tendientes a mejorar las destrezas y habilidades informativas, así como la implementación de herramientas que permitan la verificación de información consultada por la ciudadanía en plataformas o redes sociales digitales podrían coadyuvar al mejoramiento de la calidad y veracidad de la información que está generando opinión pública y participación en aspectos políticos, económicos, culturales y sociales.

Lo anterior representa un reto mayor, que al tiempo que afronta la necesidad de la puesta en marcha de esas herramientas digitales, debe ser capaz de interactuar tanto con los usuarios de la información como con otras agencias públicas, privadas y gubernamentales relacionadas con el manejo de información socialmente relevante, pues a fin de cuentas lo que está en juego es la calidad y el futuro de la democracia como sistema político incluyente y participativo.

\section{REFERENCIAS}

Álvarez, José Francisco. 2009. "Ciberciudadanía, cultura y bienes públicos”. Arbor 185 (737): 569-79. https://doi.org/10.3989/arbor.2009.1737.314

Aparicio, Javier. 2011. "En respuesta Se buscan followers”, en Ciudadanos.mx Twitter y el cambio político en México, editado por Ana Francisca Vega y José Merino, 199-207. Ciudad de México: Random House Mondadori.

Arendt, Hannah. 1993. La condición bumana. Barcelona: Paidós.

Arcila, Carlos. 2006. "El ciudadano digital". Revista Latinoamericana de Comunicación CHASQUI 93: 18-21.

Asociación de Internet.mx. 2018. "Estudio sobre los hábitos de los usuarios de Internet en México 2018”. http://www.asociaciondeinternet.org.mx/es/component/ remository/Habitos-de-Internet/lang,es-es/?Itemid=

Asociación para la Investigación de Medios de Comunicación. 2017. "Infografía Resumen 20o Navegantes en la Red”. España: Asociación para la Investigación de Medios de Comunicación. http://www.aimc.es/otros-estudios-trabajos/navegantes-la-red/infografia-resumen-20o-navegantes-la-red/

Beck, Ulrich. 1998. La sociedad del riesgo global. Barcelona: Paidós Ibérica.

Beck, Ulrich y Elisabeth Beck-Gernsheim. 2003. La individualización. El individualismo institucionalizado y sus consecuencias sociales y políticas. Barcelona: Paidós Ibérica.

Benítez, Lucía. 2013. "La dimensión transnacional de la ciudadanía digital", en Ciudadanía, tecnología y cultura. Nodos conceptuales para pensar la nueva mediación digital, editado por Francisco Sierra, 79-118. Barcelona: Gedisa. 
Bertot, John Carlo, Paul Jaeger y Charles R. McClure, eds. 2010. Public Libraries and the Internet: Roles, Perspectives, and Implications. Connecticut: Libraries Unlimited.

Bruce, Christine Susan. 1999. "Workplace experiences of information literacy”. International Journal of Information Management 19 (1): 33-47. https://doi.org/10.1016/ S0268-4012(98)00045-0

Burchardt, Hans-Jürgen. 2012. “¿Por qué América Latina es tan desigual? Tentativas de explicación desde una perspectiva inusual”. Nueva Sociedad (239): 137-50.

Burin, Debora, Yamila Coccimiglio, Federico González y Jhon Bulla. 2016. “Desarrollos recientes sobre Habilidades Digitales y Comprensión Lectora en Entornos Digitales". Psicología, Conocimiento y Sociedad 6 (1): 191-206.

Cabero, Julio. 2007. "Las necesidades de las TIC en el ámbito educativo: oportunidades, riesgos y necesidades". Tecnología y Comunicación Educativas (45): 4-19.

Campos, Eva y Aitor Silván. 2012. "Democracia digital. El estado de la cuestión”, en Ciudadania en 3D: Democracia digital deliberativa, un análisis exploratorio, editado por Irene Ramos y Eva Campos. Barcelona: Edhasa.

Castells, Manuel. 2001. La Galaxia Internet. Barcelona: Plaza y Janés.

Castells, Manuel. 2002. La Era de la Información. Vol. I: La Sociedad Red. Ciudad de México: Siglo XXI.

Castells, Manuel. 2006. La sociedad red: una visión global. Madrid: Alianza Editorial.

Cebrián, Mariano. 2009. "Comunicación interactiva en los cibermedios”. Comunicar: Revista científica iberoamericana de comunicación y educación (33): 15-24.

Dader, José Luis. 2003. "Ciberdemocracia y comunicación política virtual: el futuro de la ciudadanía electrónica tras la era de la televisión”, en Comunicación política en televisión y nuevos medios, editado por Gonzalo Salomé, 309-342. Barcelona: Ariel.

Dezuanni, Michael y Andrés Monroy. 2012. "Prosumidores interculturales: la creación de medios digitales globales entre jóvenes". Comunicar XVX (38): 59-66.

Fernández-Ramos, Andrés. 2015. "Evaluación de 'subject gateways': propuesta metodológica y análisis longitudinal”. Revista Española de Documentación Cientifica 38 (2): $1-18$.

Fleury, Sonia. 2005. "Construcción de ciudadanía en entornos de desigualdad". Instituciones y Desarrollo 16 (1): 133-170.

Griffiths, Mary. 2004. "E-Citizens: Blogging as Democratic Practice”. Electronic Journal of E-Government 2 (3): 1-10.

Habermas, Jürgen. 1987. Teoría de la acción comunicativa. Volumen 2: Crítica de la razón funcionalista. Madrid: Taurus.

Hintlin, Paul. 2018. "Use of Internet, Social Media, Digital Devices Plateau in US". Pew Research Center (blog). http://www.pewresearch.org/fact-tank/2018/09/28/ internet-social-media-use-and-device-ownership-in-u-s-have-plateaued-afteryears-of-growth/

Hoffman, Donna L., Thomas P. Novak y Ann E. Schlosser. 2001. “The evolution of the digital divide: examining the relationship of race to internet access and usage over time", en The Digital Divide, editado por Benjamin M. Compaine, 47-97. Cambridge: MIT Press. http://dl.acm.org/citation.cfm?id=762625.762630

Horrigan, John y Maeve Duggan. 2015. "Barriers to Broadband Adoption: Cost Is Now a Substantial Challenge for Many Non-Users”. Home Broaband 2015. http://www.pewinternet.org/2015/12/21/3-barriers-to-broadband-adoption-costis-now-a-substantial-challenge-for-many-non-users/ 
Islas, Octavio. 2010. "Internet 2.0: El territorio digital de los prosumidores". Revista Estudios Culturales (5): 43-63.

Kaufman, Ester. 2005. "E-Ciudadanía, Prácticas de Buen Gobierno y TIC”. Consulta Regional del Programa Pan Américas IDRC. https://idl-bnc-idrc.dspacedirect.org/ bitstream/handle/10625/55408/IDL-55408.pdf? sequence=1

Kymlicka, Will y Wayne Norman. 1997. "El retorno del ciudadano. Una revisión de la producción reciente en teoría de la ciudadanía”. Agora 7: 5-42.

La Due Lake, Ronald y Robert Huckfeldt. 1998. "Social Capital, Social Networks, and Political Participation”. Political Psychology 19 (3): 567-584. https://doi.org/10.1111/0162-895X.00118

Marcos, Juan Carlos, Juan Miguel Sánchez y María Olivera. 2018. "El consumo de información en las redes sociales: encrucijada de los medios digitales", en Conocimientos sin fronteras: Colaboración científica e institucional en Documentación e Información, editado por M. Teresa Fernández Bajón, Rodrigo Sánchez e Isabel Villaseñor, 718-741. Madrid: Universidad Complutense de Madrid.

Marshall, Thomas H. 1997. "Ciudadanía y clase social”. Reis 79: 297-344.

McClurg, Scott D. 2003. "Social Networks and Political Participation: The Role of Social Interaction in Explaining Political Participation”. Political Research Quarterly 56 (4): 449-64. https://doi.org/10.1177/106591290305600407

Miller, Mark, James Reus, Robb Matzke, Quincey Koziol y Albert Cheng. 2004. "Smart Libraries: Best SQE Practices for Libraries with an Emphasis on Scientific Computing". Trabajo presentado en Nuclear Explosives Code Developer's Conference, California, 15 de diciembre. https://www.osti.gov/biblio/936460

Monfort, Nuria. 2013. "Internet: de la rapidez a la inmediatez", adComunica 5: 269271. https://doi.org/10.6035/118

Morales Campos, Estela. 2006. Infodiversidad y cibercultura: Globalización e información en América Latina. Buenos Aires: Alfagrama Ediciones.

Natal, Alejandro, Mónica Benítez y Gladys Ortiz. 2014. Ciudadanía digital. México: Juan Pablos.

Nielsen, Jakob. 2006. "Participation inequality: Lurkers vs. contributors in internet communities". Nielsen's Alertbox (blog). https://www.nngroup.com/articles/ participation-inequality/

ONU (Organización de las Naciones Unidas). 2012. "América Latina es la Región más desigual del planeta”. América economía, sec. Economía \& Mercados. http:// www.americaeconomia.com/economia-mercados/finanzas/onu-america-latinaes-la-region-mas-desigual-del-planeta

OEA (Organización de los Estados Americanos). 2014. "OEA: "América Latina es la región más desigual del mundo” I ICNDiario”. ICN Iberoamérica Central de Noticias (blog). http://www.icndiario.com/2014/06/14/oea-america-latina-es-la-region-mas-desigual-del-mundo/

Palanque, Philippe y Fabio Paternò. 1998. "Electronic Gridlock, Information Saturation and the Unpredictability of Information Retrieval over the World Wide Web", en Formal Methods in Human-Computer Interaction, 261-282. Formal Approaches to Computing and Information Technology (FACIT). Londres: Springer. https://doi.org/10.1007/978-1-4471-3425-1_13

Pérez, Antonio-Enrique. 2014. "Teledemocracia, ciberciudadanía y derechos humanos”. Revista Brasileira de Políticas Públicas 4 (2): 8-46. 
Postmes, Tom y Suzanne Brunsting. 2002. "Collective Action in the Age of the Internet: Mass Communication and Online Mobilization”. Social Science Computer Review 20 (3): 290-301. https://doi.org/10.1177/089443930202000306

Repanovici, Angela, Mihai Turcanu, Luciana Cristea, Mihaela Baritz y Ioana Moisil. 2009. "Smart Library: RFID Implementation in Libraries", en Proceedings of the 8th WSEAS International Conference on Artificial Intelligence, Knowledge Engineering and Data Bases, 523-526. AIKED'09. Stevens Point, Wisconsin, USA: World Scientific and Engineering Academy and Society (WSEAS). http://dl.acm. org/citation.cfm?id=1553921.1554017

Ribble, Mike S., Gerald D. Bailey y Tweed W. Ross. 2004. "Digital Citizenship: Addressing Appropriate Technology Behavior". Learning E Leading with Technology 32 (1): 6.

Robles, José Manuel. 2009. Ciudadanía digital: Una introducción a un nuevo concepto de ciudadano. Barcelona: Editorial UOC.

Rodríguez, Adolfo. 2006. La brecha digital y sus determinantes. Ciudad de México: UNAM-CUIB.

Rodríguez, Mariángela. 2005. Tradición, identidad, mito y metáfora, mexicanos y chicanos en California. Ciudad de México: Porrúa/CIESAS.

Sánchez Navarro, Jordi y Daniel Aranda Juárez. 2011. "Internet como fuente de información para la vida cotidiana de los jóvenes españoles". El profesional de la información 20 (1): 32-37.

Sebestova, Eva, Jaroslav Bendl, Jan Brezovsky y Jiri Damborsky. 2014. “Computational Tools for Designing Smart Libraries”, en Directed Evolution Library Creation, editado por Frances H. Arnold y George Georgiou, 291-314. Nueva York: Springer. https://doi.org/10.1007/978-1-4939-1053-3_20

Servon, Lisa. 2002. Bridging the Digital Divide. Technology, community and public policy. Oxford: Blackwell Publishing.

Shelley, Mack, Lisa Thrane, Stuart Shulman, Evette Lang, Sally Beisser, Teresa Larson y James Mutiti. 2004. "Digital Citizenship Parameters of the Digital Divide". Social Science Computer Review 22 (2): 256-69. https://doi.org/10.1177/0894439303262580

Shields, Bob. 1996. Cultures of the Internet. Virtual Spaces, Real Histories, Living Bodies. California: Sage Publications.

Silva, Ana. 2010. “¿Ciudadanía electrónica? El gobierno electrónico desde la perspectiva de los usuarios. Una aproximación etnográfica”, en IX Cumbre Iberoamericana de Comunicadores, 1-22. San Martín: UNSAM.

Spitzer, Kathleen L., Michael B. Eisenberg y Carrie A. Lowe. 1998. Information Literacy: Essential Skills for the Information Age. Nueva York: Information Resources Publications, Syracuse University. https://eric.ed.gov/?id=ED427780

Sterret, David. 2012. "El potencial y las limitantes de internet para fomentar la deliberación y la democracia en el mundo", en Ciudadanía en 3D: Democracia digital deliberativa, un análisis exploratorio, editado por Irene Ramos y Eva Campos, 85135. Barcelona: Edhasa.

Tesich, Steve. 1992. "A Government of Lies". The Nation 6 (13): 12-14.

Torres, Gerardo. 2007. Ciudadanía y Cultura Politica (Intelectuales mexicanos de fin de siglo XIX). Toluca, México: IAPEM.

Trejo-Delarbre, Raúl. 2017. "En la era de la posverdad. La manipulación de las redes sociodigitales". TELOS Revista de Pensamiento sobre Comunicación, Tecnología y Sociedad (117): 8-10. 
Turner, Bryan S. 1990. "Outline of a Theory of Citizenship". The Journal of the British Sociological Association 24 2: 189-217. https://doi.org/10.1177/0038038590024002002

Vieira, Liszt. 1998. "Ciudadanía y control social”, en Lo público no estatal en la reforma del Estado, editado por Nuria Cunill y Luiz Carlos Bresser, 215-256. Argentina: Paidós Ibérica. http://dialnet.unirioja.es/servlet/articulo?codigo $=610311$

Vleugels, Roger. 2009. Overview of all 90 FOIA countries and territories. California: Fringe Special.

Voutssas, Juan. 2006. Bibliotecas y publicaciones digitales. México: Centro Universitario de Investigaciones Bibliotecológicas y de la Información-UNAM.

Voutssas, Juan. 2017. Confianza e información digital: bibliotecas, archivos y web. Ciudad de México: IIBI-UNAM.

Watts, Duncan J. y Peter Sheridan Dodds. 2007. "Influentials, Networks, and Public Opinion Formation”. Journal of Consumer Research 34 (4): 441-458. https://doi. org/10.1086/518527

Wellman, Barry, Anabel Quan-Haase, James Witte y Keith Hampton. 2001. "Does the Internet Increase, Decrease, or Supplement Social Capital?: Social Networks, Participation, and Community Commitment". American Behavioral Scientist 45 (3): 436-55. https://doi.org/10.1177/00027640121957286

Won Min, Byung. 2012. "Next Generation Library information service Smart Library". International Journal of Software Engineering and Its Applications 6 (4): 171-174. https://pdfs.semanticscholar.org/a060/c58e0787700e6e470e6fe8a6dd64bc3e598d.pdf

Zickuhr, Kathryn. 2013. Who's Not Online and Why I Pew Research Center. http:// www.pewinternet.org/2013/09/25/whos-not-online-and-why/

Para citar este texto:

Ramos Chávez, Héctor Alejandro. 2019. "Ciudadanía e información en ambientes digitales”. Investigación Bibliotecológica: archivonomía, bibliotecología e información 33 (78): 143-163.

http://dx.doi.org/10.22201/iibi.24488321xe.2019.78.58045 